\title{
P128 Accuracy of Age-appropriate Transfer Functions in Modelling Central Arterial Waveform Features
}

\author{
Tommy Cai ${ }^{1,2,}$, Meroni Alice ${ }^{1}$, Ahmad Qasem ${ }^{3,4}$, Mark Butlin ${ }^{3}$, Julian Ayer ${ }^{1,5}$, David Celermajer ${ }^{1,2}$, \\ Alberto Avolio ${ }^{3}$, Michael Skilton ${ }^{1}$
}

${ }^{1}$ University of Sydney, Sydney, Australia

${ }^{2}$ Royal Prince Alfred Hospital, Sydney, Australia

${ }^{3}$ Macquarie University, Sydney, Australia

${ }^{4}$ AtCor Medical, Sydney, Australia

${ }^{5}$ The Children's Hospital at Westmead, Sydney, Australia

\section{ABSTRACT}

Background: Central aortic pressure waveforms can be modelled non-invasively in adults using general transfer functions. These techniques have been applied to, but not formally validated in paediatric populations. We recently developed and validated two age-appropriate paediatric transfer functions and sought to determine their effectiveness in children and adolescents.

Methods: We recruited 97 healthy children between 2 and 20 years of age in five pre-specified age groups. Central waveforms were estimated by applying two previously developed paediatric transfer functions developed in 8 year and 14 year-old children (8TF, 14TF respectively), and a proprietary adult transfer function (aTF) (Sphygmocor CvMS, AtCor) to radial waveforms measured by tonometry [1]. Estimated central arterial parameters were measured from direct carotid tonometry.

Results: $8 \mathrm{TF}$ estimates higher cSBP than 14TF, which estimates higher cSBP than aTF across all ages. In contrast, 8TF estimates lower cAIx than 14TF, which estimates lower cAIx than aTF across all ages (Figure 1). 8TF most accurately modelled central arterial waveform features, specifically by cAIx when comparing to carotid tonometry, in early childhood (age groups: 2-6.5 years, $0 \pm 14 \%$; $6.6-9.5$ years, $-4 \pm 19 \%$; 9.6-12.5 years, $-6 \pm 13 \%$ ), while $14 \mathrm{TF}$ most accurately estimated cAIx in later childhood and adolescence (12.6-15.5 years, $-3 \pm 11 \%$; $15.6-20$ years, $4 \pm 13 \%$ ).

Conclusion: Effectiveness of transfer functions for modelling central arterial waveforms in childhood appear to be agedependent, with evidence of better accuracy when used in populations similar to that in which they were developed. These results support the use of multiple age-appropriate paediatric transfer functions over a generalized paediatric transfer function.
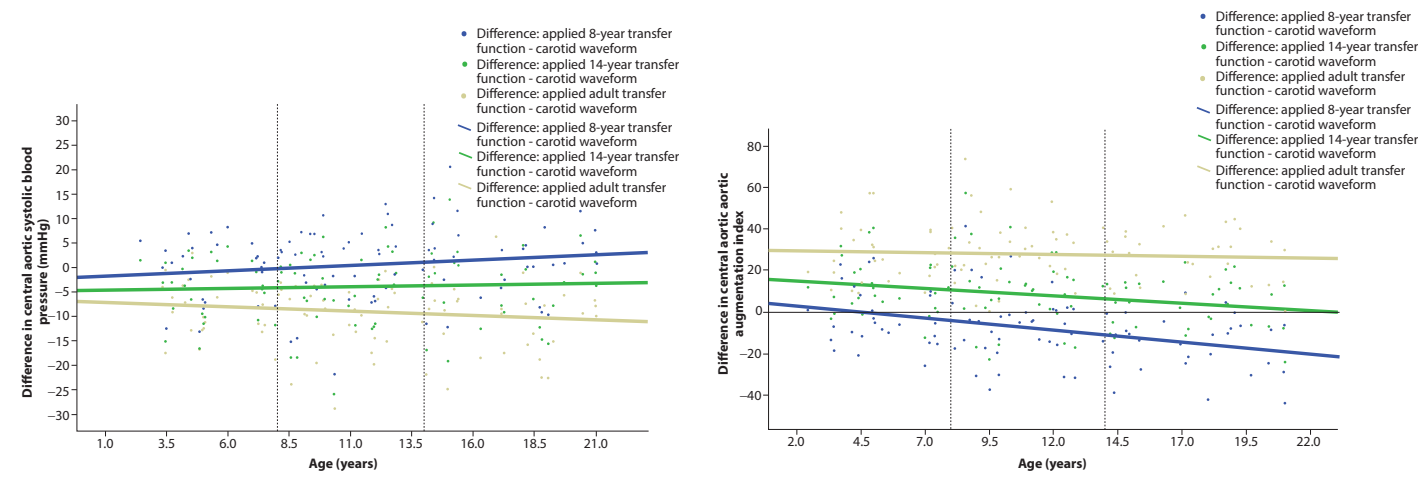

Figure 1

\section{REFERENCE}

[1] Cai TY, Qasem A, Ayer JG, Butlin M, O’Meagher S, Melki C, et al. Central blood pressure in children and adolescents: non-invasive development and testing of novel transfer functions. J Hum Hypertens 2017;31:831-37.

(c) 2019 Association for Research into Arterial Structure and Physiology. Publishing services by Atlantis Press International B.V. This is an open access article distributed under the CC BY-NC 4.0 license (http://creativecommons.org/licenses/by-nc/4.0/). 